\title{
Global risk of deadly heat
}

Article

Accepted Version

Mora, C., Dousset, B., Caldwell, I. R., Powell, F. E., Geronimo, R. C., Bielecki, C. R., Counsell, C. W. W., Dietrich, B. S., Johnston, E. T., Louis, L. V., Lucas, M. P., McKenzie, M. M., Shea, A. G., Tseng, H., Giambelluca, T. W., Leon, L. R., Hawkins, E. and Trauernicht, C. (2017) Global risk of deadly heat. Nature Climate Change, 7 (7). pp. 501-506. ISSN 1758678X doi: https://doi.org/10.1038/nclimate3322 Available at https://centaur.reading.ac.uk/71263/

It is advisable to refer to the publisher's version if you intend to cite from the work. See Guidance on citing.

Published version at: http://dx.doi.org/10.1038/nclimate3322

To link to this article DOI: http://dx.doi.org/10.1038/nclimate3322

Publisher: Nature Publishing Group

All outputs in CentAUR are protected by Intellectual Property Rights law, including copyright law. Copyright and IPR is retained by the creators or other copyright holders. Terms and conditions for use of this material are defined in the End User Agreement.

$\underline{\text { www.reading.ac.uk/centaur }}$ 
Central Archive at the University of Reading

Reading's research outputs online 
One Sentence Summary: Climatic conditions capable of exceeding human thermoregulatory capacity currently impact

5 almost one third of the world's human population each year with their occurrence projected to increase in step with $\mathrm{CO}_{2}$ emissions and be aggravated in humid tropical areas.

Camilo Mora $^{1} \uparrow$, Bénédicte Dousset ${ }^{2}$, Iain R. Caldwell ${ }^{3}$, Farrah E. Powell ${ }^{1}$, Rollan C. Geronimo ${ }^{1}$, Coral R. Bielecki ${ }^{4}$, Chelsie W. W. Counsel1 ${ }^{3}$ Bonnie S. Dietrich ${ }^{5}$, Emily T. Johnston ${ }^{4}$, Leo V Louis ${ }^{4}$, Matthew P. Lucas ${ }^{6}$, Marie M. McKenzie ${ }^{1}$, Alessandra G. Shea ${ }^{1}$, Han Tseng ${ }^{1}$, Thomas W Giambelluca ${ }^{1}$, Lisa R. Leon ${ }^{7}$, Ed Hawkins $^{8}$, Clay Trauernicht ${ }^{6}$

${ }^{1}$ Department of Geography, Hawai'i i at Mānoa, Honolulu, Hawai ‘i, 96822, USA

${ }^{2}$ Hawai $\square \mathrm{i}$ Institute of Geophysics and Planetology, University of Hawai $\square \mathrm{i}$ at Mānoa, Honolulu, Hawai`i, 96822, USA

${ }^{3}$ Hawai $\square$ i Institute of Marine Biology, University of Hawai $\square$ i at Mānoa, Kāne $\square$ ohe, Hawai' ‘i, 96744, USA

${ }^{4}$ Department of Botany, University of Hawai $\square$ i at Mānoa, Honolulu, Hawai‘i, 96822, USA

${ }^{5}$ Department of Plant and Environmental Protection Sciences, University of Hawai $\square$ i at Mānoa, Honolulu, Hawai' $i, 96822$, USA

${ }^{6}$ Department of Natural Resources and Environmental Management, University of Hawai $\square$ i at Mānoa, Honolulu, Hawai ${ }^{\circ}$, 96822, USA

${ }^{7}$ Thermal and Mountain Medicine Division, U.S. Army Research Institute of Environmental Medicine, Natick, Massachusetts 01760, USA

${ }^{8}$ National Centre for Atmospheric Science, Department of Meteorology, University of Reading, Reading, Berkshire, RG6 6BB, UK

†Corresponding author. E-mail: cmora@hawaii.edu

Climate change can increase the risk of conditions that exceed human thermoregulatory capacity (1-6). Although numerous studies report increased mortality associated with extreme heat events (1-7), quantifying the global risk of heat-related mortality remains challenging due to a lack of comparable data on heat-related deaths (2-5). Here we conducted a global analysis of documented lethal heat events to identify the climatic conditions associated with 
human death and then quantified the current and projected occurrence of such deadly climatic conditions worldwide. We reviewed papers published between 1980 and 2014, and found 783 cases of excess human mortality associated with heat from 164 cities in 36 countries. We identified a global threshold beyond which daily mean surface air temperature and relative humidity become deadly. Around $30 \%$ of the world's population is currently exposed to climatic conditions exceeding this deadly threshold for at least 20 days a year. By 2100 , this percentage is projected to increase to $\sim \mathbf{4 8} \%$ under a scenario with drastic reductions of greenhouse gas emissions and $\sim 74 \%$ under a business-as-usual scenario. An increasing threat to human life from excess heat now seems almost inevitable, but will be greatly aggravated if greenhouse gases are not considerably reduced.

Sporadic heat events, lasting days to weeks, are often related to increased human mortality $(1,2)$, raising serious concerns for human health given ongoing climate change (1-3, 8-16). Unfortunately, a number of challenges have hampered global assessments of the risk of heat-related death. First, heat illness (i.e., severe exceedance of the optimum body core temperature) is often underdiagnosed because exposure to extreme heat often results in the dysfunction of multiple organs, which can lead to misdiagnosis $(2,3,5,17)$. Second, mortality data from heat exposure are sparse and have not been analyzed in a consistent manner. Here we conducted a global survey of peer-reviewed studies on heat-related mortality to identify the location and timing of past events that caused heat-related deaths. We used climatic data during those events to identify the conditions most likely to result in human death and then quantified the current and projected occurrence of such deadly climatic conditions. Hereafter, we use "lethal" when referring to climatic conditions during documented cases of excess mortality and "deadly" when referring to climatic conditions that are projected to cause death. We make this distinction to acknowledge that climatic conditions which have killed people in the past are obviously capable of causing death but whether or not they result in human mortality in the future could be affected by adaptation. We do not quantify human deaths per se because the extent of human mortality will be considerably modified by social adaptation [e.g., use of air conditioning, early warning systems, etc. (18-20)]. Although social adaptation could reduce the exposure to deadly heat (18-20), it will not affect the occurrence of such conditions. Given the speed of climatic changes and numerous physiological constraints, it is unlikely that human physiology will evolve the necessary higher heat tolerance $(21,22)$, highlighting that outdoor conditions will remain deadly even if social adaptation is broadly implemented. Our aim is to quantify where and when deadly heat conditions occur, which in turn can provide important information on where social adaptation will likely be needed. 
We searched available online databases for peer-reviewed publications on heat-related mortality published between 1980 and 2014 (see methods). From over 30,000 relevant references, we identified 911 papers that included data on 1,949 case studies of cities or regions where excess mortality was associated with high temperatures. Case studies were broadly grouped into those focusing on temperature-mortality relationships in a specific city, region, or country $(1,166$ cases from 273 cities across 49 countries) and those focusing on heat-related mortality during specific episodes (783 cases from 164 cities across 36 countries). Cases were predominantly reported for cities at mid-latitudes, with the highest concentration in North America and Europe (Fig. 1a), and included well-documented heatwaves like those in Chicago in 1995 ( 740 deaths, 23), Paris in 2003 ( 4,870 deaths, 24), Moscow in 2010 ( $\sim 10,860$ deaths, 25$)$ and many other, less publicized events (list of cases provided at https://rollan.shinyapps.io/HeatwavesMap/). While data on the number of deaths was inconsistently reported, all studies provided information on the place and dates when climatic conditions were lethal, which we used to identify the specific climatic conditions resulting in heat-related mortality.

To identify the climatic conditions related to lethal heat events, we assessed daily climatic data (i.e., surface air temperature, relative humidity, solar radiation, wind speed, and several other metrics, Fig. S1) for the duration of lethal heat episodes reported in the literature and an equal number of non-lethal episodes (i.e., periods of equal duration from the same cities but from randomly selected dates); then we used Support Vector Machines (SVM) to identify the climatic conditions that best differentiated lethal and non-lethal episodes. SVMs generate a threshold that maximizes the difference in the attributes of two or more groups allowing to classify objects in either group based on where their given attributes fall with respect to the threshold. In our case, SVM was used to generate a decision threshold that maximizes the difference in climatic conditions of lethal and non-lethal episodes with the conditions on one side of the threshold being lethal and those to the other side being non-lethal (e.g., Fig. 1b). Among all possible pair combinations of the variables analyzed here (Fig. S1-S2), the SVM using mean daily surface air temperature and relative humidity most accurately distinguished between past lethal and non-lethal heat episodes (i.e., $82 \%$, blue line in Fig. 1b); accuracy was measured as the ratio of the number of correctly classified lethal and non-lethal cases to the total number of cases. Adding other variables to the temperaturehumidity SVM resulted in less parsimonious SVMs with minimal increases in accuracy (e.g., the SVM model including all 16 variables analyzed here was only $3 \%$ more accurate, Fig. S3). SVM also allows to estimate a classification probability that increases with the distance of an observation to the decision threshold; the use of a $95 \%$ probability for the temperaturehumidity SVM (red line in Fig. 1b) resulted in 100\% accurate predictions of true positives (i.e., only prior lethal heat episodes were on the deadly side of the $95 \%$ probability SVM decision boundary). While our analysis used data on local 
climatic conditions, the resulting pattern between temperature and relative humidity allowed us to accurately classify lethal heat events of different cities from around the world using a single common SVM threshold (Fig. 1b).

The fact that temperature and relative humidity best predict times when climatic conditions become deadly is consistent with human thermal physiology, as they are both directly related to body heat exchange (2-4). First, the combination of an optimum body core temperature (i.e., $\left.\sim 37^{\circ} \mathrm{C}\right)$, the fact that our metabolism generates heat $(\sim 100 \mathrm{~W}$ at rest) and that an object cannot dissipate heat to an environment with equal or higher temperature (i.e. the second law of thermodynamics, 22), dictates that any ambient temperature above $37^{\circ} \mathrm{C}$ should result in body heat accumulation and a dangerous exceedance of the optimum body core temperature [hyperthermia (5)]. Second, sweating, the main process by which the body dissipates heat, becomes ineffective at high relative humidity (i.e., air saturated with water vapor prevents evaporation of sweat); therefore, body heat accumulation can occur at temperatures lower than the optimum body core temperature in environments of high relative humidity. These properties help to explain why the boundary at which temperature becomes deadly decreases with increasing relative humidity (Fig. 1b) and why in our results some heat mortality events occurred at relatively low temperatures (Fig. 1b). Both, temperature and relative humidity are included in traditional thermal indexes such as humidex (26) and wet-bulb globe temperature $(22,27)$.

To quantify the global extent of current deadly climatic conditions, we applied the $95 \%$ probability SVM decision boundary between mean daily surface air temperature and relative humidity (red line in Fig. 1b, hereafter referred to as deadly threshold) to current global climate data (see Methods). Using data from a climate reanalysis (see methods), we found that in $2000, \sim 13.2 \%$ of the planet's land area, where $\sim 30.6 \%$ of the world's human population resides, was exposed to 20 or more days when temperature and humidity surpassed the threshold beyond which such conditions become deadly (Fig. 2, extended results in Fig. S4). Comparatively, using climate simulations for the year 2000 (i.e., historical experiment) developed for the Coupled Model Intercomparison Project phase 5 (CMIP5), we found that $\sim 16.2 \%(+/-8.3 \%$ standard deviation, SD) of the planet's land area, where $\sim 37.0 \%$ (+/-9.7\% SD) of the world's population resides, was exposed to 20 or more days of potentially deadly conditions of temperature and humidity (results are multimodel medians and standard deviations among Earth System Models; Fig. 2). Both the re-analysis and historical CMIP5 data revealed increasing trends in the area and population exposed to deadly climates during the time period for which such datasets can be compared although the trends in the reanalysis are slightly weaker than in the ESMs (Fig. 2). Overall, there was $\sim 3 \%$ mismatch in the area of the planet ( $\sim 6.4 \%$ in global population) between the reanalysis and the multimodel median, and thus, results based on CMIP5 simulations should be interpreted with that error in mind. However, the effects of this mismatch and the uncertainty among Earth System Models were smaller than the predicted changes in deadly days (Fig. S10). It is worth 
noticing that most scientific report on deadly heat events have occurred in developed mid-latitude countries (Fig. 1a); yet, deadly heat conditions also occur in developing tropical countries (Fig. 3). This suggests the possibility that the risk of deadly heat could be currently underestimated in tropical regions, which has been noted in prior studies (28).

To predict the global extent of future deadly climates, we applied the deadly SVM threshold to mean daily surface air temperature and relative humidity projections from the CMIP5 Earth System Models under low, moderate, and high emissions scenarios (Representative Concentration Pathways, RCPs, 2.6, 4.5, and 8.5, respectively). We found that by 2100, even under the most aggressive mitigation scenario (i.e., RCP 2.6$), \sim 26.9 \%(+/-8.7 \%$ SD) of the world's land area will be exposed to temperature and humidity conditions exceeding the deadly threshold by more than 20 days per year, exposing $\sim 47.6 \%$ (+/-9.6\% SD) of the world's human population to deadly climates (using Shared Socioeconomic Pathways of human population (29) relevant to each of the CMIP5 RCPs, see methods). Scenarios with higher emissions will affect an even greater percentage of the global land area and human population. By $2100, \sim 34.1 \%(+/-7.6 \% \mathrm{SD})$ and $\sim 47.1 \%(+/-$ $8.9 \% \mathrm{SD}$ ) of the global land area will be exposed to temperature and humidity conditions that exceed the deadly threshold for more than 20 days per year under RCP 4.5 and RCP 8.5 , respectively; this will expose $\sim 53.7 \%(+/-8.7 \%$ SD) and $\sim 73.9 \%(+/-6.6 \% \mathrm{SD})$ of the world's human population to deadly climates by the end of the century (Fig. 2, extended results in Fig. S4).

The projected number of days per year surpassing the deadly threshold increases from mid-latitudes to the equator (Fig. 5a, Fig S5a,d,g). By 2100 , for example, mid-latitudes (e.g., $40^{\circ} \mathrm{N}$ or S) will be exposed to $\sim 60$ deadly days per year compared to almost the entire year in humid tropical areas under RCP 8.5 (Fig. 3b-d, Fig. 4b-d, Fig. 5a). This latitudinal pattern was consistent among all scenarios (Fig. S5a,d,g) and is largely determined by the fact that the number of days with temperatures close to the deadly threshold declines with increasing latitude [i.e., due to greater seasonality; Fig. S6b-d, (28)]. For example, at mid-latitudes (e.g., New York, Fig. 4i-l) temperatures only approach the deadly threshold during the summer, which represents a smaller proportion of the year; compared to tropical locations (e.g., Jakarta, Fig. 4e-h), which have consistently warm temperatures near the deadly threshold year-round (Fig. S6). Although tropical humid areas will experience less warming than higher latitudes (Fig. 5b, see also 30), they will be exposed to the greatest increase in the number of deadly days over time, because higher relative humidity in tropical areas requires lower temperatures to cross the deadly threshold (Fig. 4e-h, 5e); a condition that could be further aggravated by projected increases in relative humidity of tropical areas (Fig. 5a). Sub-tropical and mid-latitude areas will have fewer days beyond the deadly threshold, but such days will be much hotter in the future (Fig. 4e-h, 5b,d). This general variability in the climatic conditions of deadly days (Fig. 5bd, Fig. S7) is likely related to mean global climate patterns associated with the general circulation of the atmosphere: 
equatorial convection (i.e., warm, moist air rising) produces high humidity in low latitudes whereas subtropical atmospheric

145 subsidence (i.e., cool, dry air sinking) creates low-precipitation, low-humidity zones, where high sensible heat flux

146 contributes to extreme high temperatures, at mid-latitudes (Fig. S5i, Fig. S7).

147 Our study underscores the current and increasing threat to human life posed by climate conditions that exceed

148 human thermo regulatory capacity. Lethal heatwaves are often mentioned as a key consequence of ongoing climate change,

149 with reports typically citing past major events such as Chicago 1995, Paris 2003, or Moscow 2010 (1-6). Our literature

150 review indicates, however, that lethal heat events already occur frequently and in many more cities worldwide than

151 suggested by these highly cited examples. Our analysis shows that prior lethal events occurred beyond a general threshold

152 of combined temperature and humidity and that today nearly one-third of the world's population is regularly exposed to

153 climatic conditions surpassing this deadly threshold. The area of the planet and fraction of the world's human population

154 exposed to deadly heat will continue to increase under all emission scenarios, although the risk will be much greater under

155 higher emission scenarios. By 2100, almost three-quarters of the world's human population could be exposed to deadly

156 climatic conditions under high future emissions (RCP 8.5) as opposed to one-half under strong mitigation (RCP 2.6). While

157 it is understood that higher latitudes will undergo more warming than tropical regions (30), our results suggest that tropical

158 humid areas will be disproportionately exposed to more days with deadly climatic conditions (Fig. 5a), because these areas

159 have year-round warm temperatures and higher humidity, thus requiring less warming to cross the deadly threshold (Fig. 4,

160 S6). The consequences of exposure to deadly climatic conditions could be further aggravated by an aging population (i.e., a

161 sector of the population highly vulnerable to heat; 2, 3, 4) and increasing urbanization (i.e., exacerbating heat-island effects;

$1622,3,4)$. Our paper emphasizes the importance of aggressive mitigation to minimize exposure to deadly climates and

163 highlights areas of the planet where adaptation will be most needed.

164

165 1. J. A. Patz, D. Campbell-Lendrum, T. Holloway, J. A. Foley, Impact of regional climate change on human health. $166 \quad$ Nature 438, 310 (2005).

167 2. R. Basu, J. M. Samet, Relation between elevated ambient temperature and mortality: a review of the epidemiologic 168 evidence. Epidemiol. Rev. 24, 190 (2002).

169 3. R. S. Kovats, S. Hajat, Heat stress and public health: A critical review. Annu. Rev. Publ. Health 29, 41 (2008).

170 4. L. R. Leon, Pathophysiology of heat stroke. Colloquium Series on Integrated Systems Physiology: From Molecule to 171 Function to Disease (Morgan \& Claypool Life Sciences, 2015), vol. 7, pp. 1-101.

172 5. B. D. Ostro, L. A. Roth, R. S. Green, R. Basu, Estimating the mortality effect of the July 2006 California heat wave. 173 Environ. Res. 109, 614 (2009).

174 6. J. Glaser et al., Climate change and the emergent epidemic of chronic kidney disease from heat stress in rural 175 communities: The case for heat stress nephropathy. Clin. J. Am. Soc. Nephrol., (2016). 
7. J.-M. Robine et al., Death toll exceeded 70,000 in Europe during the summer of 2003. C. R. Biol. 331, 171 (2008).

8. J. Sillmann, E. Roeckner, Indices for extreme events in projections of anthropogenic climate change. Clim. Chang. 86, 83 (2008).

9. G. A. Meehl, C. Tebaldi, More intense, more frequent, and longer lasting heat waves in the 21 st century. Science 305, 994 (2004).

10. B. Orlowsky, S. Seneviratne, Global changes in extreme events: regional and seasonal dimension. Clim. Change 110, $669(2012 / 02 / 01,2012)$.

11. C. Tebaldi, K. Hayhoe, J. M. Arblaster, G. A. Meehl, Going to the extremes. Clim. Change 79, 185 (2006).

12. C. Tebaldi, M. F. Wehner, Benefits of mitigation for future heat extremes under RCP4. 5 compared to RCP8. 5. Clim. Change, 1 (2016).

13. A. Sterl et al., When can we expect extremely high surface temperatures? Geophys. Res. Lett. 35, L14703 (2008).

14. C. Huang et al., Projecting future heat-related mortality under climate change scenarios: a systematic review. Environ. Health Persp. 119, 1681 (2011).

15. Y. Guo et al., Global variation in the effects of ambient temperature on mortality: a systematic evaluation. $J$. Epidemiol. 25, 781 (2014).

16. G. Luber, M. McGeehin, Climate change and extreme heat events. Am. J. Prev. Med. 35, 429 (2008).

17. A. Bouchama, J. P. Knochel, Heat stroke. New. Engl. J. Med. 346, 1978 (2002).

18. J. F. Bobb, R. D. Peng, M. L. Bell, F. Dominici, Heat-related mortality and adaptation to heat in the United States. Environ. Health Persp. 122, 811 (2014).

19. A. Gasparrini et al., Temporal variation in heat-mortality associations: a multicountry study. Environ. Health Persp. 123, 1200 (2015).

20. D. Lowe, K. L. Ebi, B. Forsberg, Heatwave early warning systems and adaptation advice to reduce human health consequences of heatwaves. Int. J. Environ. Res. Public Health 8, 4623 (2011).

21. E. G. Hanna, P. W. Tait, Limitations to thermoregulation and acclimatization challenge human adaptation to global warming. Int. J. Environ. Res. Publ. Health. 12, 8034 (2015).

22. S. C. Sherwood, M. Huber, An adaptability limit to climate change due to heat stress. Proc. Natl. Acad. Sci. USA 107, 9552 (2010).

23. S. Whitman et al., Mortality in Chicago attributed to the July 1995 heat wave. Am. J. Public Health 87, 1515 (1997).

24. B. Dousset et al., Satellite monitoring of summer heat waves in the Paris metropolitan area. Int. J. Climatol. 31, 313 (2011).

25. D. Shaposhnikov et al., Mortality related to air pollution with the Moscow heat wave and wildfire of 2010. Epidemiol. 25, 359 (2014).

26. A. G. Barnett, S. Tong, A. Clements, What measure of temperature is the best predictor of mortality? Environ. Res. 110, 604 (2010).

27. K. M. Willett, and Steven Sherwood, Exceedance of heat index thresholds for 15 regions under a warming climate using the wet-bulb globe temperature. Int. J. Climatol. 32, 161 (2012).

28. D. Argüeso, A. Di Luca, S. Perkins-Kirkpatrick, J. P. Evans, Seasonal mean temperature changes control future heatwaves. Geophys.

Res. Lett., 7653 (2016). 
29. B. Jones, B. O’Neill, Spatially explicit global population scenarios consistent with the Shared Socioeconomic Pathways. Environ. Res. Lett. 11, 084003 (2016).

30. N. S. Diffenbaugh, C. B. Field, Changes in ecologically critical terrestrial climate conditions. Science $\mathbf{3 4 1}, 486$ (2013).

31. D. Mitchell et al., Attributing human mortality during extreme heat waves to anthropogenic climate change. Environ. Res. Lett. 11, 074006 (2016).

Supplementary Information is available in the online version of the paper.

Acknowledgments We thank the Gridded Human Population of the World Database and the National Center for Environmental Prediction and Department of Defense reanalysis database for making their data openly available and Bryan Jones for sharing human population projections. We acknowledge the World Climate Research Programme's Working Group on Coupled Modelling, which is responsible for CMIP5, and thank the climate modeling groups (listed in Table S1) for producing and making available their model outputs. We also thank David Schanzenbach, Sean Cleveland and Ron Merrill from the University of Hawaii Super Computer Facility for allowing access to computing facilities and SeaGrant Hawaii for providing funds to acquire some of the computers used in these analyses. Qi Chen, Adam Smith, Chelsea Dau, Rong Fang, and Sonia Seneviratne provided valuable contributions to the paper. The opinions or assertions contained herein are the private views of the authors and are not to be construed as official or as reflecting the views of the Army or the Department of Defense. This paper was developed as part of the graduate course on "Methods for Large-Scale Analyses" in the Department of Geography, University of Hawaii at Manoa.

Authors Contributions All authors contributed to the design of the paper. CM, BD, IRC, FEP, RCG, CRB, CWWC, BSD, ETJ, LVL, MPL, MMM, AGS, HT and CT collected data. CM, IRC performed analysis. All authors contributed to the writing of the paper.

\section{Figure captions}

Fig. 1. Geographical distribution of recent lethal heat events and their climatic conditions. a, places where relationships between heat and mortality have been documented (red squares) and where specific heat episodes have been studied (black crosses). b, mean daily surface air temperature and relative humidity during lethal heat events (black crosses) and during periods of equal duration from the same cities but from randomly selected dates (i.e., non-lethal heat events; red to yellow gradient indicates the density of such non-lethal events). Blue line is the SVM threshold that best separates lethal and non-lethal heat events and the red line is the 95\% probability SVM threshold; areas to the right of the thresholds are classified as deadly and those to the left as non-deadly. Support vectors for other variables are shown in Fig. S2.

Fig. 2. Current and projected changes in deadly climatic conditions. a, area of the planet and b, human population exposed to climatic conditions beyond the 95\% SVM deadly threshold (red line in Fig. 1b) for at least 20 days in a year under alternative emission scenarios. Bolded lines are the multimodel medians, black lines are the results from re-analysis data and faded lines indicate the projections for each Earth System Model. Time series were smoothed with a 10 year average moving window. Area of the planet and human population exposed to different lengths of time are shown in Fig. 
S4. Results correcting for climatological mean biases between the re-analysis data and each Earth System Model are shown in Fig. S8,S10.

Fig. 3. Geographical distribution of deadly climatic conditions under different emission scenarios. Number of days per year exceeding the threshold of temperature and humidity beyond which climatic conditions become deadly (Fig. 1b), averaged between 1995 and 2005 (a, historical experiment) and between 2090 and 2100 under RCP 2.6 (b), RCP 4.5 (c), and RCP 8.5 (d). Results are based on multi-model medians. Grey areas indicate locations with high uncertainty (i.e., the multimodel standard deviation was larger than the projected mean; coefficient of variance $>1$ ). The expected lower number of deadly days at higher latitudes (Fig. 4) may help explain the large variability among Earth System Models in the projected number of deadly days at higher latitudes (31) [e.g., in the case for New York (illustrated in Fig 4.j) the one model projects nine deadly days by 2100 ; yet any other model projecting 18 days will double the variability]. The uncertainty presented in this figure should be interpreted with that caution in mind.

266

Fig. 4. Latitudinal risk of deadly climates. a-d, distribution of the percentage of days in a given year (i.e., color gradients), at each latitude, as a function of their distance to the deadly threshold (red line in Fig. 1b). Displayed here are the last year in the historical experiment (i.e., 2005; a) and the year 2100 under RCP 2.6 (b), RCP 4.5 (c) and RCP 8.5 (d).

270 These plots illustrate that higher latitudes have fewer days near the deadly threshold compared to the tropics. As examples, we show mean temperature and relative humidity for each day in the year 2005 in the historical experiments and the year 2100 for all the RCPs in Jakarta (e-h) and New York (i-l), with consecutive days connected by lines. The 95\% SVM threshold is shown as a red line with numbers on the upper right hand corner indicating the number of days that cross the threshold and the difference in temperature between 2100 and 2005. Examples are based on a single simulation of a randomly chosen model (i.e., CSIRO-Mk3-6-0). over time in the number of days per year exceeding the deadly threshold, (b) changes in temperature and (c) changes in relative humidity during those deadly days, relative to mean value between 1995 and 2005. Plots $\mathbf{d}$ and e show the mean temperature and relative humidity during deadly days, respectively. Results are grouped by latitude and are based on the multimodel medians for the historical experiment, which runs from 1950 to 2005, and RCP 8.5, which runs from 2006 to 2100. Results for all scenarios are shown in Fig. S5. 


\section{Methods}

Survey of published cases of heat-related mortality. We searched for peer-reviewed studies published between 1980 and 2014 on heat-related mortality in Google Scholar, PubMed, and the Web-of-Science using the following keywords: (human OR people) AND (mortality OR death OR lethal) AND (heat OR temperature). We searched for papers primarily in English but also included papers in Spanish, French, Japanese, and Chinese when found. We reviewed the titles and abstracts of the first 30,000 citations in Google Scholar and all citations from other databases and selected any peer-reviewed publications on heat-related human mortality (we also searched for additional sources in the references). These efforts resulted in 911 peer-reviewed papers from which we collected information on the place and dates of lethal heat events. Several papers noted that human mortality may have occurred beyond the dates in which the extreme climatic conditions occurred ("mortality displacement"); in those cases, we extracted the dates for which the extreme climatic conditions were reported in the given studies. Our goal was to identify the dates in which climatic conditions triggered human mortality regardless of whether mortality was lagged or not.

Climatic conditions related to prior cases of heat related mortality. For the cases in the literature review that reported the place and time of lethal heat events, we assessed information for 16 climatic metrics based on mean daily surface air temperature, relative humidity, solar radiation, and wind speed (Fig. S1). For each of the lethal heat events, we also assessed the same climatic variables for a paired "non-lethal" event of the same duration and from the same city but from a randomly chosen date. Climatic conditions were characterized using daily data from an atmospheric reanalysis of past climate (NCEPDOE Reanalysis 2). We used the NCEP-DOE Reanalysis database because it is among the most studied and is well characterized relative to newer databases. We used Support Vector Machine (SVM) modeling to separate the climatic conditions associated with prior lethal heat events from those associated with non-lethal events. Using SVM, we generated a decision vector/threshold that maximized the distance between lethal and non-lethal episodes with the conditions on one side of the threshold being lethal and those to the other side being non-lethal (e.g., Fig. 1b). We developed such SVM models for all combinations of the variables collected and then compared the accuracy of models to choose the most parsimonious and best performing one.

Projected occurrence of deadly climatic conditions. To quantify the number of days in a year that surpass the threshold beyond which conditions become deadly under alternative emission scenarios, we applied the $95 \%$ SVM probability threshold between mean daily surface air temperature and relative humidity of prior lethal heat events to daily climate 
projections of the same variables. We used the $95 \%$ SVM probability threshold because it resulted in a much more accurate classification of prior lethal heat events, and because it restricts projected lethal heat events to much more extreme conditions, hence yielding more conservative results. We used daily climate projections of mean surface air temperature and relative humidity from 20 Earth System Models under four alternative emissions scenarios developed for the recent Coupled Model Intercomparison Project Phase 5 (Table S1). We used the 'historical' experiment, which includes the period from 1950 to 2005 and the Representative Concentration Pathways 2.6, 4.5 and 8.5 (RCP 2.6, 4.5 and 8.5, respectively), which include the period from 2006 to 2100 . The historical experiment was designed to model recent climate (reflecting changes due to both anthropogenic and natural causes) and allows the validation of model outputs against available climate observations (Fig. S8-9). RCP pathways represent contrasting mitigation efforts between rapid greenhouse gas reductions (RCP 2.6) and a business-as-usual scenario (RCP 8.5). All analyses were run at the original resolution of each climate database and the results were interpolated to a common $1.5^{\circ}$ grid cell size using a bilinear function.

\section{Projections of global land coverage and risk to human populations from deadly climatic conditions. To calculate the} amount of land area and fraction of the human population that are likely to be exposed to deadly climates each year, we summed the land area and human population for all cells experiencing varying numbers of days in a year beyond the deadly threshold (Fig. 2, Fig. S4). We used the Gridded Population of the World from the Socioeconomic Data and Applications Center (http://sedac.ciesin.columbia.edu/data/set/gpw-v3-population-count-future-estimates/data-download\#) to estimate human exposure up to the year 2005 and human population projections consistent with the different emission scenarios used in the CMIP5 to estimate exposure between 2006 and 2100. For the population projections, we specifically used the spatially explicit global population scenarios consistent with the Shared Socioeconomic Pathways (SSP) developed by Jones et al (29), pairing RCP 2.6 with SSP1, RCP 4.5 with SSP3, and RCP 8.5 with SSP5.

\section{Limitations}

There are several potential limitations to our study. First, the lethality of deadly climatic conditions can be mediated by various demographic (e.g., age structure), socio-economic (e.g., air conditioning, early warning systems) and urban planning (e.g., vegetation, high albedo surface) factors that were not considered in our study. Consideration of these factors would improve the understanding of global human vulnerability to heat exposure and may reduce the number of human deaths, but they are unlikely to affect the occurrence of deadly climatic conditions, which is what we estimated. Second, our survey of cases of heat related mortality was restricted to the period between 1980 and 2014 and any bias or temporal 
341 heterogeneity in the monitoring of lethal heatwaves and epidemiological studies in this period may influence the cases we

342 studied and the resulting SVM model. Third, while general agreement among models was found in the predictions of deadly

343 climatic conditions in tropical areas, greater variability among models was seen in such projections at higher latitudes (grey

344 areas in Fig. 3). Because deadly conditions are more rare at higher latitudes (Fig. 4), a larger number of model ensembles

345 might allow for more definitive statements about the risk of deadly climates in such regions, as has been suggested for

346 similar cases of rare events (3131). Finally, it is possible that some lethal heat events were not documented in peer-reviewed

347 publications and, if the dates of those undocumented events happened to be selected as part of the non-lethal events in our

348 analysis, this could affect the resulting SVM model. However, this error is likely minimal because there is a low probability

349 of randomly selecting such rare and brief events from a 30 year period in the given cities.

350

\section{Data availability}

352 The data that support the findings of this study are available from the corresponding author upon request. 